Vol. 49 (1994) [267-275]

\title{
MINIMAX INEQUALITIES AND GENERALISATIONS OF THE GALE-NIKAIDO-DEBREU LEMMA
}

\author{
KOK-KEONg TAN AND JiAN YU
}

\begin{abstract}
Some minimax inequalities are first proved both in the compact case and in the non-compact case using the concept of escaping sequences introduced by Border. Applications are given to deduce a generalisation of the Gale-Nikaido-Debreu Lemma due to Mehta and Tarafdar and to obtain a new generalisation of the Gale-Nikaido-Debreu Lemma from which the corresponding generalisation due to Grandmont is derived.
\end{abstract}

\section{INTRODUCTION}

The Gale-Nikaido-Debreu Lemma (in short, the GND Lemma), see $[4,6,12]$ (see also [2, Theorem 18.1, p.81]), is fundamental to proving the existence of a market equilibrium of an economy, for example, see Border [2] and Debreu [5]. Recently, there have been many generalisation of this Lemma, see $[7,8,10,11,14]$. The objective of this paper is two-fold:

(1) we first give some minimax inequalities both in the compact case and in the non-compact case using the concept of escaping sequences introduced by Border [2];

(2) as applications of the minimax inequalities,

(a) we deduce a generalisation of the Gale-Nikaido-Debreu Lemma due to Mehta and Tarafdar [10] which in turn generalises that of Yannelis [14] and

(b) we obtain a new generalisation of the Gale-Nikaido-Debreu Lemma, from which the corresponding generalisation due to Grandmont [8] is derived.

\section{Preliminaries}

If $A$ is a set, $2^{A}$ denotes the family of all subsets of $A$. If $A$ is a subset of a vector space, coA denotes the convex hull of $A$. We shall denote by $\mathbb{R}$ and $\mathbb{N}$ the set of all real numbers and the set of all natural numbers respectively. If $A$ is a non-empty subset of $\mathbb{R}^{m}$ and $p \in \mathbb{R}^{m}$, dist $(p, A)$ denotes the distance from $p$ to A. If $p=\left(p_{1}, \cdots, p_{m}\right) \in \mathbb{R}^{m}$, then $p \geqslant 0$ (respectively, $\left.p>0, p \leqslant 0\right)$ if $p_{i} \geqslant 0$

Received 19th April, 1993

Copyright Clearance Centre, Inc. Serial-fee code: 0004-9729/94 SA2.00+0.00. 
(respectively, $p_{i}>0, p_{i} \leqslant 0$ ) for all $i=1, \cdots, m$. If $X$ and $Y$ are topological spaces and $T: X \rightarrow 2^{Y}$, then $T$ is upper semicontinuous if for each open subset $U$ of $Y$, the set $\{x \in X: T(x) \subset U\}$ is open in $X$. If $X$ is a non-empty convex subset of a vector space, then $f: X \rightarrow \mathbb{R}$ is quasi-concave (respectively, quasi-convex) if for each $\lambda \in \mathbb{R}$, the set $\{x \in X: f(x)>\lambda\}$ (respectively, the set $\{x \in X: f(x)<\lambda\}$ ) is convex.

\section{Generalisations of the GND Lemma: The compact case}

We first prove the following minimax inequality:

THEOREM 1. Let $X$ be a non-empty compact convex subset of a Hausdorff topological vector space $E$ and let $Y$ be a non-empty convex subset of a Hausdorff topological vector space $F$. Suppose that the real-valued function $f: X \times Y \rightarrow \mathbb{R}$ and the set-valued map $T: X \rightarrow 2^{Y}$ satisfy the following conditions:

(i) for each fixed $y \in Y, x \rightarrow f(x, y)$ is upper semicontinuous and quasiconcave (respectively, $x \rightarrow f(x, y)$ is concave);

(ii) for each fixed $x \in X, y \rightarrow f(x, y)$ is lower semicontinuous and quasiconvex (respectively, $y \rightarrow f(x, y)$ is lower semicontinuous and convex);

(iii) for each $x \in X, T(x)$ is non-empty compact, convex and $\min _{y \in T(x)} f(x, y) \leqslant$ 0 ;

(iv) for each $x \in X$ with $\left\{u \in X: \min _{y \in T(x)} f(u, y)>0\right\} \neq \emptyset$, there is $\bar{x} \in X$ such that $x \in \operatorname{int}\left\{v \in X: \min _{y \in T(v)} f(\bar{x}, y)>0\right\}$.

Then there exists $\left(x^{*}, y^{*}\right) \in X \times Y$ with $y^{*} \in T\left(x^{*}\right)$ such that $f\left(x, y^{*}\right) \leqslant 0$ for all $x \in X$.

Proof: Define the set-valued map $F: X \rightarrow 2^{Y}$ by $F(x)=\{u \in X:$ $\left.\min _{y \in T(x)} f(u, y)>0\right\}$ for each $x \in X$. Fix an $x \in X$. For any $u_{1} \in F(x)$ and $u_{2} \in F(x)$, by (ii), (iii), there exist $y_{1} \in T(x)$ and $y_{2} \in T(x)$ such that $f\left(u_{1}, y_{1}\right)=\min _{y \in T(x)} f\left(u_{1}, y\right)>$ 0 and $f\left(u_{2}, y_{2}\right)=\min _{y \in T(x)} f\left(u_{2}, y\right)>0$. For any $\alpha \in[0,1]$, there exists $y_{\alpha} \in T(x)$ such that $f\left(\alpha u_{1}+(1-\alpha) u_{2}, y_{\alpha}\right)=\min _{y \in T(x)} f\left(\alpha u_{1}+(1-\alpha) u_{2}, y\right)$. By (i),

$$
\begin{aligned}
f\left(\alpha u_{1}+(1-\alpha) u_{2}, y_{\alpha}\right) & \geqslant \min \left\{f\left(u_{1}, y_{\alpha}\right), f\left(u_{2}, y_{\alpha}\right)\right\} \\
& \geqslant \min \left\{f\left(u_{1}, y_{1}\right), f\left(u_{2}, y_{2}\right)\right\}>0
\end{aligned}
$$

so that $\alpha u_{1}+(1-\alpha) u_{2} \in F(x)$. Thus $F(x)$ is convex for each $x \in X$.

By (iii), $x \notin F(x)$ for all $x \in X$.

For each $x^{\prime} \in X, F^{-1}\left(x^{\prime}\right)=\left\{v \in X: \min _{y \in T(v)} f\left(x^{\prime}, y\right)>0\right\}$. By (iv), if $F(x) \neq 0$, then there is $\bar{x} \in X$ such that $x \in$ int $F^{-1}(\bar{x})$. 
Theorem 4 of $[10]$ implies that there exists $x^{*} \in X$ such that $F\left(x^{*}\right)=0$, that is, $\min _{y \in T\left(x^{*}\right)} f(u, y) \leqslant 0$ for all $u \in X$.

Now, since conditions (i), (ii) and (iii) hold, by [13, Theorem 3.4] (respectively by [7, Corollary $9.4(\mathrm{~b})]$ ), we have

$$
\min _{y \in T\left(x^{*}\right)} \max _{x \in X} f(x, y)=\max _{x \in X} \min _{y \in T\left(x^{*}\right)} f(x, y) \leqslant 0
$$

(respectively, $\min _{y \in T\left(x^{*}\right)} \sup _{x \in X} f(x, y)=\sup _{x \in X} \min _{y \in T\left(x^{*}\right)} f(x, y) \leqslant 0$ ).

Hence there exists $y^{*} \in F\left(x^{*}\right)$ such that $\max _{x \in X} f\left(x, y^{*}\right) \leqslant 0$ (respectively, $\sup _{x \in X} f\left(x, y^{*}\right)$ $\leqslant 0)$, that is, $f\left(x, y^{*}\right) \leqslant 0$ for all $x \in X$.

As an application of Theorem 1, we have the following generalisation of the GaleNikaido-Debreu Lemma due to Mehta and Tarafdar [10, Theorem 8]:

Corollary 1. Let $E$ be a real Hausdorff locally convex topological vector space, $E^{*}$ be the topological dual of $E$ equipped with the weak topology, $C$ be a closed convex cone of $E$ having an interior point $e, C^{*}=\left\{p \in E^{*}:\langle p, y\rangle \leqslant 0\right.$ for all $y \in C\} \neq\{0\}$ be the dual cone of $C$, and $\Delta=\left\{p \in C^{*}:\langle p, e\rangle=-1\right\}$. Suppose that the set-valued map $T: \Delta \rightarrow 2^{E}$ satisfies the following conditions:

(i) for each $p \in \Delta, T(p)$ is non-empty compact convex and $\min _{y \in T(p)}\langle p, y\rangle \leqslant 0$;

(ii) for each $p \in \Delta$ with $\left\{q \in \Delta: \min _{y \in T(p)}\langle q, y\rangle>0\right\} \neq 0$, there is $\bar{p} \in \Delta$ such that $p \in \operatorname{int}\left\{\bar{q} \in \Delta: \min _{y \in T(\bar{q})}\langle\bar{p}, y\rangle>0\right\}$.

Then there exists $p^{*} \in \Delta$ such that $T\left(p^{*}\right) \cap C \neq \emptyset$.

Proof: Set $X=\Delta$, then $X$ is convex. Since $\Delta$ is equicontinuous and $w^{*}$-closed, the Alaoglu theorem [9, Theorem 3.8, p.123] implies that it is $w^{*}$-compact.

Set $Y=E$ and $f(p, y)=\langle p, y\rangle$, then the conditions of Theorem 1 hold so that there exist $p^{*} \in \Delta$ and $y^{*} \in T\left(p^{*}\right)$ such that $\left\langle p, y^{*}\right\rangle \leqslant 0$ for all $p \in \Delta$.

We shall prove that $y^{*} \in C$ and hence $y^{*} \in T\left(p^{*}\right) \cap C$.

If $y^{*} \notin C$, since $E$ is locally convex and $C$ is closed convex, by [3, p.111, Corollary $3.10]$, there exists $r \in E^{*}$ with $r \neq 0$ such that $\sup _{y \in C}\langle r, y\rangle\left\langle\left\langle r, y^{*}\right\rangle\right.$. Since $0 \in C$ and $C$ is a cone, we must have $\sup _{y \in C}\langle r, y\rangle=0$. It follows that $r \in C^{*}$ and $\left\langle r, y^{*}\right\rangle>0$. Since $e \in \operatorname{int} C$, we have $r(e)<0$. Let $\bar{r}=-(r / r(e))$, then $\bar{r} \in \Delta$. But $\left\langle\bar{r}, y^{*}\right\rangle>0$ which contradicts the fact that $\left\langle p, y^{*}\right\rangle \leqslant 0$ for all $p \in \Delta$. Hence we must have $y^{*} \in C$.

By [10, Remark 1] Corollary 1 is more general than Theorem 3.1 of [14]. Hence Theorem 1 also generalises Theorem 3.1 of [14].

In what follows we deduce another minimax inequality from Theorem 1. 
THEOREM 2. Let $X$ be a non-empty compact convex subset of a Hausdorff topological vector space $E$, and let $Y$ be a non-empty convex subset of a Hausdorff topological vector space $F$. Suppose that the real-valued function $f: X \times Y \rightarrow \mathbb{R}$ and the set-valued map $T: X \rightarrow 2^{Y}$ satisfy the following conditions:

(i) for each fixed $y \in Y, x \rightarrow f(x, y)$ is upper semicontinuous and quasiconcave (respectively, $x \rightarrow f(x, y)$ is concave);

(ii) for each fixed $x \in X, y \rightarrow f(x, y)$ is lower semicontinuous and quasiconvex (respectively, $y \rightarrow f(x, y)$ is lower semicontinuous and convex);

(iii) for each $x \in X, T(x)$ is non-empty compact convex and $\min _{y \in T(x)} f(x, y) \leqslant$ 0 ;

(iv) for each $x \in X,\left\{u \in X: \min _{y \in T(u)} f(x, y) \leqslant 0\right\}$ is closed.

Then there exists $\left(x^{*}, y^{*}\right) \in X \times Y$ with $y^{*} \in T\left(x^{*}\right)$ such that $f\left(x, y^{*}\right) \leqslant 0$ for all $x \in X$.

Proof: We only need to prove that the condition (iv) of Theorem 1 holds: Indeed, let $x \in X$ be such that $\left\{u \in X: \min _{y \in T(x)} f(u, y)>0\right\} \neq \emptyset$ and take $\bar{x} \in\{u \in X$ : $\left.\min _{y \in T(x)} f(u, y)>0\right\}$, that is, $\min _{y \in T(x)} f(\bar{x}, y)>0$. Thus $x \notin\left\{u \in X: \min _{y \in T(u)} f(\bar{x}, y) \leqslant\right.$ $0\}$. Since $\left\{u \in X: \min _{y \in \Gamma(u)} f(\bar{x}, y) \leqslant 0\right\}$ is closed, it follows that $x \in \operatorname{int}\{u \in X$ : $\left.\min _{y \in T(u)} f(\bar{x}, y)>0\right\}$.

As an application of Theorem 2, we derive the following minimax inequality due to Granas and Liu [7, Theorem (13.1)]:

COROLlary 2. Let $X$ be a non-empty compact convex subset of a Hausdorff topological vector space $E$, and let $Y$ be a non-empty convex subset of a Hausdorff topological vector space $F$. Let $T: X \rightarrow 2^{Y}$ be upper semicontinuous with non-empty compact convex values and $g: X \times Y \rightarrow \mathbb{R}$ satisfy one of the following conditions:

$\left\{\begin{array}{l}\text { For each fixed } y \in Y, \quad x \rightarrow g(x, y) \text { is lower semicontinuous } \\ \text { and quasi-convex, } \\ \text { for each fixed } x \in X, \quad y \rightarrow g(x, y) \text { is upper semicontinuous } \\ \text { and quasi-concave. }\end{array}\right.$

$$
\left\{\begin{array}{l}
\text { For each fixed } y \in Y, \quad x \rightarrow g(x, y) \text { is convex; } \\
\text { for each fixed } x \in X, \quad y \rightarrow g(x, y) \text { is upper semicontinuous } \\
\text { and concave. }
\end{array}\right.
$$

Then for each $\lambda \in \mathbb{R}$, one of the following properties holds:

(A) there exists $\bar{x} \in X$ such that $\max _{y \in T(\bar{x})} g(\bar{x}, y)<\lambda$; 
(B) there exists $\left(x^{*}, y^{*}\right) \in X \times Y$ with $y^{*} \in T\left(x^{*}\right)$ such that $\min _{x \in X} g\left(x, y^{*}\right) \geqslant \lambda$.

Proof: Assume that (I) (respectively, (II)) holds. If $(A)$ is not true, then for each $x \in X, \max _{y \in T(x)}[g(x, y)-\lambda] \geqslant 0$. Define $f: X \times Y \rightarrow \mathbb{R}$ by $f(x, y)=\lambda-g(x, y)$ for each $(x, y) \in X \times Y$. Then the conditions (i), (ii) and (iii) of Theorem 2 hold. Now fix an $x \in X$. Define $W: X \times Y \rightarrow \mathbb{R}$ by $W(u, y)=g(x, y)-\lambda$ for each $(u, y) \in X \times Y$. Then $W$ is upper semicontinuous. Since $T$ is upper semicontinuous such that for each $u \in X, T(u)$ is compact, by $[1$, p.52, Theorem 5], the map $V: X \rightarrow \mathbb{R}$ defined by $V(u)=\max _{y \in T(u)}[g(x, y)-\lambda]$ for each $u \in X$ is upper semicontinuous. It follows that the set $\left\{u \in X: \min _{y \in T(u)} f(x, y) \leqslant 0\right\}=\{u \in X: V(u) \geqslant 0\}$ is closed. Thus the condition (iv) of Theorem 2 also holds. Hence there exists $\left(x^{*}, y^{*}\right) \in X \times Y$ with $y^{*} \in T\left(x^{*}\right)$ such that $f\left(x, y^{*}\right) \leqslant 0$ for all $x \in X$, that is, $\min _{x \in X} g(x, y) \geqslant \lambda$.

The following simple example shows that Theorem 2 is a true generalisation of $[7$, Theorem (13.1)].

Example 1. Let $E=F=\mathbb{R}, X=[0, \pi / 2]$ and $Y=\mathbb{R}$. Define $g: X \times Y \rightarrow \mathbb{R}$ by $g(x, y)=y-\sin x$ for all $(x, y) \in X \times Y$. Then for each fixed $y \in Y, x \rightarrow g(x, y)$ is continuous and convex and for each fixed $x \in X, y \rightarrow g(x, y)$ is continuous and concave. Define $T: X \rightarrow 2^{Y}$ by

$$
T(x)= \begin{cases}{\left[\frac{1}{2} \sin x, \sin x\right],} & \text { if } x \neq 0, \\ \{1\}, & \text { if } x=0\end{cases}
$$

for each $x \in X$. Then $T$ has non-empty compact convex values but $T$ is not upper semicontinuous at $x=1$ so that [7, Theorem (13.1)] is not applicable. However, if we let $f=-g$, then we have $\min _{y \in T(x)} f(x, y) \leqslant 0$ for each $x \in X$. We shall show that for each $x \in X$, the set $\left\{u \in X: \min _{y \in T(u)} f(x, y)>0\right\}$ is open in $X$. Indeed, let $x \in X$ be arbitrarily fixed. If $u \in X$ is such that $\min _{y \in T(u)} f(x, y)>0$, we must have $0<u<x$. Let $\delta>0$ be such that $(u-\delta, u+\delta) \subset(0, x)$. It follows that for each $v \in(u-\delta, u+\delta)$, $\min _{y \in T(v)} f(x, y)=\sin x-\sin v>0$. This shows that the set $\left\{u \in X: \min _{y \in T(u)} f(x, y)>0\right\}$ is open in $X$ so that the set $\left\{u \in X: \min _{y \in T(u)} f(x, y) \leqslant 0\right\}$ is closed in $X$. Therefore Theorem 2 is applicable.

\section{Generalisations of the GND Lemma: The non-compact case}

We need the concept of an escaping sequence introduced in $[2$, p.34]: Let $X$ be a topological space such that $X=\bigcup_{n=1}^{\infty} C_{n}$ where $\left\{C_{n}\right\}_{n=1}^{\infty}$ is an increasing sequence of 
non-empty compact sets. Then a sequence $\left\{x_{n}\right\}_{n=1}^{\infty}$ in $X$ is said to be escaping from $X$ (relative to $\left\{C_{n}\right\}_{n=1}^{\infty}$ ) if for each $n \in \mathbb{N}$, there exists $M \in \mathbb{N}$ such that $y_{k} \notin C_{n}$ for all $k \geqslant M$.

We shall prove the following minimax inequality on a non-compact set.

THEOREM 3. Let $X$ be a non-empty subset of a Hausdorff topological vector space $E$ such that $X=\bigcup_{n=1}^{\infty} C_{n}$ where $\left\{C_{n}\right\}_{n=1}^{\infty}$ is an increasing sequence of non-empty compact convex subsets of $X$, and let $Y$ be a non-empty convex subset of a Hausdorff topological vector space $F$. Suppose that the real-valued function $f: X \times Y \rightarrow \mathbb{R}$ and the set-valued map $T: X \rightarrow 2^{Y}$ satisfy the following conditions:

(i) for each fixed $y \in Y, x \rightarrow f(x, y)$ is upper semicontinuous and quasiconcave (respectively, $x \rightarrow f(x, y)$ is concave);

(ii) for each fixed $x \in X, y \rightarrow f(x, y)$ is lower semicontinuous and quasiconvex (respectively, $y \rightarrow f(x, y)$ is lower semicontinuous and convex);

(iii) for each $x \in X, T(x)$ is non-empty compact convex and $\min _{y \in T(x)} f(x, y) \leqslant$ 0 ;

(iv) for each $n \in \mathbb{N}$ and each $x \in C_{n}$ with $\left\{u \in C_{n}: \min _{y \in T(x)} f(u, y)>0\right\} \neq 0$, there is $\bar{x} \in C_{n}$ such that $x \in \operatorname{int}\left\{v \in C_{n}: \min _{y \in T(v)} f(\bar{x}, y)>0\right\}$;

(v) for each sequence $\left\{x_{n}\right\}_{n=1}^{\infty}$, where $x_{n} \in C_{n}$ for each $n=1,2, \cdots$, which is escaping from $X$ relative to $\left\{C_{n}\right\}_{n=1}^{\infty}$ and each sequence $\left\{y_{n}\right\}_{n=1}^{\infty}$, where $y_{n} \in T\left(x_{n}\right)$ for each $n=1,2, \cdots$, there exist $n_{0} \in \mathbb{N}$ and $x_{n_{0}}^{\prime} \in C_{n_{0}}$ with $f\left(x_{n_{0}}^{\prime}, y_{n_{0}}\right)>0$.

Then there exists $\left(x^{*}, y^{*}\right) \in X \times Y$ with $y^{*} \in T\left(x^{*}\right)$ such that $f\left(x, y^{*}\right) \leqslant 0$ for all $x \in X$.

Proof: For each $n \in \mathbb{N}$ by Theorem 1 , there exists $\left(x_{n}, y_{n}\right) \in C_{n} \times Y$ with $y_{n} \in T\left(x_{n}\right)$ such that $f\left(x, y_{n}\right) \leqslant 0$ for all $x \in C_{n}$.

Suppose that the sequence $\left\{x_{n}\right\}_{n=1}^{\infty}$ were escaping from $X$ relative to $\left\{C_{n}\right\}_{n=1}^{\infty}$. By (v), there exist $n_{0} \in N$ and $x_{n_{0}}^{\prime} \in C_{n_{0}}$ with $f\left(x_{n_{0}}^{\prime}, y_{n_{0}}\right)>0$ which is a contradiction. Therefore the sequence $\left\{x_{n}\right\}_{n=1}^{\infty}$ is not escaping from $X$ relative to $\left\{C_{n}\right\}_{n=1}^{\infty}$, so that some subsequence of $\left\{x_{n}\right\}_{n=1}^{\infty}$ must lie entirely in some $C_{n_{1}}$. Since $C_{n_{1}}$ is compact, there exist a subnet $\left\{z_{\alpha}\right\}_{\alpha \in \Gamma}$ of $\left\{x_{n}\right\}_{n=1}^{\infty}$ in $C_{n_{1}}$ and $x^{*} \in C_{n_{1}}$ such that $z_{\alpha} \rightarrow x^{*}$. Let $z_{\alpha}=x_{n(\alpha)}$ where $n(\alpha) \rightarrow \infty$.

If $\left\{u \in X: \min _{y \in T\left(x^{*}\right)} f(u, y)>0\right\} \neq 0$, there exists $n_{2} \geqslant n_{1}$ such that $\{u \in$ $\left.C_{n_{2}}: \min _{y \in T\left(x^{*}\right)} f(u, y)>0\right\} \neq 0$. By (iv), there is $\bar{x} \in C_{n_{2}}$ such that $x^{*} \in \operatorname{int}\{v \in$ $\left.C_{n_{2}}: \min _{y \in T(v)} f(\bar{x}, y)>0\right\}$. Since $z_{\alpha} \rightarrow x^{*}$, there is $\alpha_{0}$ such that $n\left(\alpha_{0}\right) \geqslant n_{2}$ and $\min _{y \in T\left(z_{0}\right)} f(\bar{x}, y)>0$, hence $f\left(\bar{x}, y_{n\left(\alpha_{0}\right)}\right)>0$. This contradicts the fact that 
$\bar{x} \in C_{n\left(\alpha_{0}\right)}$ and $f\left(\bar{x}, y_{n\left(\alpha_{0}\right)}\right) \leqslant 0$. Therefore $\left\{u \in X: \min _{y \in T\left(x^{*}\right)} f(u, y)>0\right\}=\emptyset$, that is, $\min _{y \in T\left(x^{*}\right)} f(u, y) \leqslant 0$ for all $u \in X$.

By [13, Corollary 3.5$]$ (respectively, by $[7$, Corollary 9.4 (b)]), we have

$$
\min _{y \in T\left(x^{*}\right)} \sup _{x \in X} f(x, y)=\sup _{x \in X} \min _{y \in T\left(x^{*}\right)} f(x, y) \leqslant 0 .
$$

Hence there exists $y^{*} \in T\left(x^{*}\right)$ such that $\sup _{x \in X} f\left(x, y^{*}\right) \leqslant 0$, that is, $f\left(x, y^{*}\right) \leqslant 0$ for all $x \in X$.

As an application of Theorem 3, we obtain the following generalisation of the GaleNikaido-Debreu Lemma:

Theorem 4. Let $\Delta=\left\{p \in \mathbb{R}^{m}: p \geqslant 0, \sum_{i=1}^{m} p_{i}=1\right\}, S=\left\{p \in \mathbb{R}^{m}: p>\right.$ $\left.0, \sum_{i=1}^{m} p_{i}=1\right\}$ and $C_{n}=\operatorname{co}\{p \in S: \operatorname{dist}(p, \Delta \backslash S) \geqslant 1 / n\}$ for $n=1,2, \cdots$. Suppose that the set-valued map $T: S \rightarrow 2^{R^{m}}$ satisfies the following conditions:

(i) $T$ is upper semicontinuous such that for each $p \in S, T(p)$ is non-empty compact convex;

(ii) for each $p \in S$ and each $y \in T(p),\langle p, y\rangle=0$;

(iii) for each sequence $\left\{p_{n}\right\}_{n=1}^{\infty}$, where $p_{n} \in C_{n}$ for each $n=1,2, \cdots$, which is escaping from $X$ relative to $\left\{C_{n}\right\}_{n=1}^{\infty}$ and for each sequence $\left\{y_{n}\right\}_{n=1}^{\infty}$, where $y_{n} \in T\left(p_{n}\right)$ for each $n=1,2, \cdots$, there exist $n_{0} \in \mathbb{N}$ and $p_{n_{0}}^{\prime} \in$ $C_{n_{0}}$ with $\left\langle p_{n_{0}}^{\prime}, y_{n_{0}}\right\rangle>0$.

Then there exists $p^{*} \in S$ such that $0 \in T\left(p^{*}\right)$.

Proof: The conclusion clearly holds for $m=1$. Now suppose that $m>1$. We may assume that each $C_{n}$ is non-empty. Note that each $C_{n}$ is compact and convex and $S=\bigcup_{n=1}^{\infty} C_{n}$. Set $X=S$ and $Y=\mathbb{R}^{m}$. Let $f: X \times Y \rightarrow \mathbb{R}$ be defined by $f(p, y)=\langle p, y\rangle$ for each $(p, y) \in X \times Y$. Then similar to the proof of Corollary 2, we can prove that for each $n \in \mathbb{N}$ and each $p \in C_{n}$, the set $\left\{u \in C_{n}: \min _{y \in T(u)} f(p, y) \leqslant 0\right\}$ is closed. Also, similar to the proof of Theorem 2, the condition (iv) of Theorem 3 holds. By Theorem 3 , there exists $\left(p^{*}, y^{*}\right) \in S \times Y$ with $y^{*} \in T\left(p^{*}\right)$ such that $\left\langle p, y^{*}\right\rangle \leqslant 0$ for all $p \in S$.

If $y^{*} \leqslant 0$ does not hold, there is $i \in\{1, \cdots, m\}$ such that $y_{i}^{*}>0$. We choose $a$ with $0<a<1$ such that

$$
\frac{1-a}{m-1} \sum_{\substack{j \neq i \\ 1 \leqslant j \leqslant m}} y_{j}^{*}+a y_{i}^{*}>0 .
$$


Let $p_{i}=a, p_{j}=(1-a) /(m-1)(j \neq i)$. Then $p \in S$ and $\left\langle p, y^{*}\right\rangle>0$, which is impossible. Thus we must have $y^{*} \leqslant 0$. On the other hand, since $p^{*} \in S$ and $\left\langle p^{*}, y^{*}\right\rangle=0$, we obtain $y^{*}=0$ and hence $0 \in T\left(p^{*}\right)$.

Finally, we shall deduce the following generalisation of the Gale-Nikaido-Debreu Lemma due to Grandmont [8, Lemma 1]:

Corollary 3. Let $\Delta=\left\{p \in \mathbb{R}^{m}: p \geqslant 0, \sum_{i=1}^{m} p_{i}=1\right\}$ and $S=\left\{p \in \mathbb{R}^{m}: p>\right.$ $\left.0, \sum_{i=1}^{m} p_{i}=1\right\}$. Suppose that the set-valued map $T: S \rightarrow 2^{\mathbb{R}^{m}}$ satisfies the following conditions:

(i) $T$ is upper semicontinuous such that for each $p \in S, T(p)$ is non-empty compact convex;

(ii) for each $p \in S$ and each $y \in T(p),\langle p, y\rangle=0$;

(iii) for each sequence $\left\{p_{n}\right\}_{n=1}^{\infty}$ in $S$ with $p_{n} \rightarrow p \in \Delta \backslash S$ and each sequence $\left\{y_{n}\right\}_{n=1}^{\infty}$, where $y_{n} \in T\left(p_{n}\right)$ for each $n=1,2, \cdots$, there is $\bar{p} \in S$ such that $\left\langle\bar{p}, y_{n}\right\rangle>0$ for infinitely many $n$.

Then there exists $p^{*} \in S$ such that $0 \in T\left(p^{*}\right)$.

Proof: We shall show that the condition (iii) of Theorem 4 holds. Indeed, let $\left\{p_{n}\right\}_{n=1}^{\infty}$ be a sequence, where $p_{n} \in C_{n}=\operatorname{co}\{p \in S: \operatorname{dist}(p, \Delta \backslash S) \geqslant 1 / n\}$ for $n=$ $1,2, \cdots$, which is escaping from $S$ relative to $\left\{C_{n}\right\}_{n=1}^{\infty}$ and let $\left\{y_{n}\right\}_{n=1}^{\infty}$ be another sequence, where $y_{n} \in T\left(p_{n}\right)$ for $n=1,2, \cdots$. Since $\left\{p_{n}\right\}_{n=1}^{\infty}$ is a sequence in $\Delta$ and $\Delta$ is compact, without loss of generality, we may suppose that $p_{n} \rightarrow p^{*} \in \Delta \backslash S$. By (iii), there is $\bar{p} \in S$ such that $\left\langle\bar{p}, y_{n}\right\rangle>0$ for infinitely many $n$. Since $S=\bigcup_{n=1}^{\infty} C_{n}$, there is $n_{1} \in \mathbb{N}$ such that $\bar{p} \in C_{n}$ for all $n \geqslant n_{1}$. Choose any $n_{0} \geqslant n_{1}$ such that $\left\langle\bar{p}, y_{n_{0}}\right\rangle>0$. The condition (iii) of Theorem 4 holds so that the conclusion follows.

By [2, p.86, Remark 18.15], the hypotheses of [8, Lemma 1] are weaker than the hypotheses of [11, Lemma 2]. Hence Theorem 4 also generalises [11, Lemma 2].

\section{REFERENCES}

[1] J.P. Aubin and A. Cellina, Differential inclusion (Springer-Verlag, Berlin, Heidelberg, New York, 1984).

[2] K.C. Border, Fixed point theorems with applications to economics and game theory (Cambridge University Press, Cambridge, 1985).

[3] J.B. Conway, A course in functional analysis (Springer-Verlag, Berlin, Heidelberg, New York, 1990).

[4] G. Debreu, 'Market equilibrium', Proc. Nat. Acad. Sci. 42 (1956), 876-878. 
[5] G. Debreu, 'Existence of competitive equilibrium,', in Handbook of Mathematical Economics II, (K.J. Arrow and M.D. Intriligator, Editors) (North-Holland, Amsterdam, 1982), pp. 697-743.

[6] D. Gale, 'The law of supply and demand', Math. Scand. 3 (1955), 155-169.

[7] A. Granas and F.-C. Liu, 'Coincidences for set-valued maps and minimax inequalities', $J$. Math. Pures Appl. 65 (1986), 119-148.

[8] J.M. Grandmont, 'Temporary general equilibrium theory', Econometrica 45 (1977), 535-572.

[9] G. Jameson, Ordered linear spaces (Springer-Verlag, Berlin, Heidelberg, New York, 1970).

[10] G. Mehta and E. Tarafdar, 'Infinite-dimensional Gale-Nikaido-Debreu theorem and a fixed point theorem of Tarafdar', J. Econom. Theory 41 (1987), 333-339.

[11] W. Neuefeind, 'Notes on existence of equilibrium proofs and boundary behavior of supply', Econometrica 48 (1980), 1831-1837.

[12] H. Nikaido, 'On the classical multilateral exchange problem', Microeconomica 8 (1956), 135-145.

[13] M. Sion, 'On general minimax theorems', Pacific J. Math. 8 (1958), 171-176.

[14] N.C. Yannelis, 'On a market equilibrium theorem with an infinite number of commodities', J. Math. Anal. Appl. 108 (1985), 595-599.

Department of Mathematics, Statistics and Computing Science

Dalhousie University

Halifax, Nova Scotia

Canada B3H 3J5
Instituet of Applied Mathernatics

Guizhou Institute of Technology

Guiyang, Guizhou

China 550003 